\title{
Introduction to the HICSS-51 Organizational Issues of Business Intelligence, Business Analytics and Big Data Minitrack
}

\author{
Olivera Marjanovic \\ University of Sydney \\ Australia \\ olivera.marjanovic@, \\ sydney.edu.au
}

\author{
Barbara Dinter \\ Chemnitz University of \\ Technology, Germany \\ barbara.dinter@wirtschaft.tu- \\ chemnitz.de
}

\author{
Thilini Ariyachandra \\ Xavier University \\ USA \\ ariyachandrat@xavier.edu
}

New developments and the ongoing evolution of data and technologies in business intelligence (BI), business analytics (BA) and big data create exciting opportunities for innovation and progress as well as brand new challenges for individuals, organizations and society.

This minitrack, now in its $28^{\text {th }}$ year, continues its tradition as the longest running HICSS minitrack related to $\mathrm{BI}$ and $\mathrm{BA}$ and more recently big data. While BI/BA technologies and applications are rapidly changing, the minitrack remains focused on the organizational issues of $\mathrm{BI} / \mathrm{BA}$ implementations that are now more challenging than ever. This particular focus, as well as its long history of research embedded in Decision Support Systems and other research predecessors of contemporary BI/BA, creates a minitrack with a rich comprehensive view of the current and emerging organizational issues.

This year's minitrack includes the following three research papers:

- The opening paper by Goul, Mishra and Dnyanmothe investigates the innovative concept of fog computing platforms, focusing on the contract provision in Smart City infrastructures. Using complex adaptive systems theory, the authors simulate different contract provision scenarios and investigate patterns that emerge. With fog computing rapidly becoming a natural space for Internet of Things (IoT) applications, this paper opens up an exciting new research direction for this minitrack and $\mathrm{BI} / \mathrm{BA}$ research in general.

- Sternkopf and Mueller remind us of the importance of "doing good with data". Their paper describes a data literacy maturity model (DLMM) that was developed in the context of nongovernmental organizations (NGOs). The main contribution of this action design research is in the proposed data literacy maturity grid, accompanied by a self-assessment tool.
- In the final paper, Lennerholt, van Laere and Soderstrom provide a literature review of the main implementation challenges of self service BI. The authors identify and discuss ten challenges of the Self Service Business Intelligence (SSBI) approach, including the six challenges related to "Access and use of data" and further four challenges related to "Self-reliant users". This research is expected to help practitioners avoid common pitfalls when implementing self service BI as well as guide future research in this area.

We trust that you will find this year's selection of very diverse papers interesting and relevant. We also hope that you will continue to support this longest running minitrack on BI, BA and Big data at HICSS for many years to come. 